\title{
Genetic analyses of the genus Nocardioides and related taxa based on 16S-235 rDNA internally transcribed spacer sequences
}

\author{
Jung-Hoon Yoon, ${ }^{1,2}$ Sung Taik Lee ${ }^{2}$ and Yong-Ha Park ${ }^{1}$
}

Author for correspondence: Yong-Ha Park. Tel: +82428604620 . Fax: +82428604625 . e-mail: yhpark@kribb4680.kribb.re.kr

\author{
1 Korean Collection for Type \\ Cultures (KCTC), Korea \\ Research Institute of \\ Bioscience and \\ Biotechnology (KRIBB), PO \\ Box 115, Yusong, Taejon, \\ Korea \\ 2 Department of Biological \\ Sciences, Korea Advanced \\ Institute of Science and \\ Technology, Taejon, Korea
}

The 16S-23S internally transcribed spacer (ITS) sequences were analysed to clarify inter- and intraspecific relationships among strains of the genus Nocardioides and the relationship between two Aeromicrobium species. The 165-23S ITS regions from 33 Nocardioides strains, two Aeromicrobium species and Terrabacter tumescens were sequenced directly after polymerase chain reaction (PCR) amplification and $\lambda$ exonuclease treatment. The genomes of some Nocardioides strains included two types of 165-23S ITS sequences. The sizes of the 165-235 ITS sequences of Nocardioides strains ranged from 328 to 539 bp. The 16S-23S ITS sequences of Aeromicrobium erythreum NSP37', Aeromicrobium fastidiosum NSP38' and T. tumescens NSP39' were 349, 355 and 386 bp long, respectively. Nucleotide similarity among 16S-23S ITS sequences of Nocardioides albus strains and of Nocardioides simplex strains was $84.1-100 \%$ and $97 \cdot 7-100 \%$, respectively. The $165-235$ ITS sequence of Nocardioides luteus was identical to that of 'Nocardioides fulvus' NSP32' and was only 1 bp different from that of 'Nocardioides flavus' strains. However, the 16S-23S ITS sequences of ' $N$. fulvus' NSP33 showed only a low degree of similarity to ' $N$. fulvus' NSP32' $(54.8 \%)$. The degree of $165-235$ ITS similarity between $N$. luteus NSP20' and $N$. albus strains ranged from 85 to $93 \%$. The mean nucleotide similarity values between the type strains of validly described Nocardioides species were highly divergent at $68 \cdot 1 \pm 16.8 \%$. The two Aeromicrobium species showed a level of $165-235$ ITS similarity of $71 \cdot 2 \%$. In this study, 16S-23S ITS sequences of the members of the genera Nocardioides and Aeromicrobium were useful for inferring the relationships between closely related strains and species. However, they were not found to be appropriate for elucidating the phylogenetic relationships between distantly related organisms at the genus level.

Keywords: Nocardioides, rDNA, internally transcribed spacer

\section{INTRODUCTION}

The genus Nocardioides was proposed by Prauser (1976) for nocardioform actinomycetes containing LLdiaminopimelic acid, alanine, glutamic acid and glycine in the cell wall peptidoglycan (wall chemotype I; see Lechevalier \& Lechevalier, 1970). The original type species of the genus is Nocardioides albus (Prauser,

Abbreviation: ITS, internally transcribed spacer.

The GenBank accession numbers for the 165-23S ITS sequences reported in this paper are AF017470-AF017510 (see Table 1).
1976). This organism is Gram-positive, non-acid-fast, aerobic and mesophilic and forms a mycelium that fragments into irregular rod- to coccus-like elements (Prauser, 1976). Several Nocardioides species, such as Nocardioides jensenii, Nocardioides plantarum and Nocardioides simplex, which have subsequently been reclassified and identified as members of the genus Nocardioides, do not form mycelia (O'Donnell et al., 1982; Suzuki \& Komagata, 1983; Collins et al., 1989, 1994). The morphological heterogeneity found in the genus Nocardioides makes it necessary to use chemical markers to characterize the genus. The chemical markers that characterize the genus Nocardioides have 
been described elsewhere (Prauser, 1976; O'Donnell et al., 1982; Miller et al., 1991; Collins et al., 1994; Tamura \& Yokota, 1994; Yoon et al., 1997a).

There are currently five validly described Nocardioides species, namely $N$. albus (Prauser, 1976), N. luteus (Prauser, 1984), N. jensenii (Suzuki \& Komagata, 1983; Collins et al., 1989), N. plantarum (Collins et al., 1994) and N. simplex (O'Donnell et al., 1982; Suzuki \& Komagata, 1983). In addition, there are three invalidly described Nocardioides species, namely 'Nocardioides flavus' (Ruan \& Zhang, 1979), 'Nocardioides fulvus' (Ruan \& Zhang, 1979) and "Nocardioides thermolilacinus' (Lu \& Yan, 1983). The two strains of ' $N$. thermolilacinus' display a Streptomyces-like life cycle, susceptibility to Streptomyces phages and lack of susceptibility to Nocardioides phage and are, hence, regarded as streptomycetes (Prauser, 1989). Our recent $16 \mathrm{~S}$ rDNA sequence analysis also showed that the two strains are members of the genus Streptomyces (Yoon et al., 1998). Nocardioides fastidiosa (Collins \& Stackebrandt, 1989) has recently been transferred to the genus Aeromicrobium as Aeromicrobium fastidiosum based on chemotaxonomic characterization and the 16S rDNA sequence (Tamura \& Yokota, 1994).

The genus Aeromicrobium was proposed by Miller et al. (1991) for Arthrobacter sp. NRRL B-3381 (French et al., 1970). This organism differs in many ways from Arthrobacter globiformis, the type species of the genus Arthrobacter, and was therefore reclassified as a new taxon, the genus Aeromicrobium as Aeromicrobium erythreum sp. nov., based on additional taxonomic data (Miller et al., 1991). The second Aeromicrobium species is Aeromicrobium fastidiosum (Tamura \& Yokota, 1994). Thus, there are currently two Aeromicrobium species. The genus Aeromicrobium has a cell wall type based on LL-diaminopimelic acid (wall chemotype I; see Lechevalier \& Lechevalier, 1970). The additional chemical markers that characterize the genus Aeromicrobium have been described elsewhere (Miller et al., 1991; Tamura \& Yokota, 1994). The genus Aeromicrobium has been included in the family Nocardioidaceae together with the genus Nocardioides, the type genus of the family (Stackebrandt et al., 1997).

Recently, 16S rDNA sequence analysis has shown that $N$. luteus is closely related to the $N$. albus strains (Yoon et al., 1998). N. luteus 16S rDNA exhibits $100 \%$ similarity to N. albus strain JCM 5854 and $99.0-99.9 \%$ similarity to the other $17 \mathrm{~N}$. albus strains in the region corresponding to positions $28-1524$ by comparison with 16S rRNA of Escherichia coli (Brosius et al., 1991). According to 16S rRNA or rDNA sequences and DNA-DNA reassociation data (Stackebrandt \& Goebel, 1994), strains belonging to the same species normally exhibit similarity levels of $16 \mathrm{~S}$ rRNA or rDNA of more than $97 \%$. Thus, taxonomic relationships between $N$. albus strains and $N$. luteus should be re-evaluated in detail. Moreover, ' $N$. flavus' strains IFO $14396^{\mathrm{T}}$ and IFO 14397 and ' $N$. fulvus' JCM $3335^{\mathrm{T}}$ show $100 \% 16 \mathrm{~S}$ rDNA similarity to $N$. luteus. ' $N$. flavus' IFO $14396^{\mathrm{T}}$ has been assumed to be a synonym for N. luteus (Prauser, 1989), but the taxonomic position of ' $N$. fulvus' strains has not been clearly defined. The mean intraspecific $16 \mathrm{~S}$ rDNA similarities of $N$. albus and $N$. simplex were $99.5 \pm 0.5 \%$ and $100 \%$, respectively. Nocardioides-like isolate NSP41 has been shown to be related to $N$. simplex with a $16 \mathrm{~S}$ rDNA similarity value of $98.6 \%$. However, $16 \mathrm{~S}$ rDNA similarity of $100 \%$ found between existing $N$. simplex strains makes the identification of isolate NSP41 problematic. Although DNA-DNA relatedness constitutes the most important criterion for determining the exact taxonomic relationships between closely related strains and species (Stackebrandt \& Goebel, 1994), additional taxonomic markers also may be necessary for polyphasic taxonomic studies (Vandamme et al., 1996).

16S rDNA and 16S rRNA sequencing is a powerful method for elucidating phylogenetic relationships among prokaryotic organisms (Woese, 1987; Stackebrandt et al., 1997) and has been used to determine inter- and intraspecific relationships (Yoon et al., 1998). Nevertheless, 16S rDNA/16S rRNA sequences may be insufficient to define phylogenetic relationships among closely related species and among strains belonging to a species because of the evolutionary conservation of the molecule (Woese, 1987). In this study, we determined the nucleotide sequences of $16 \mathrm{~S}-23 \mathrm{~S}$ rDNA $(16 \mathrm{~S}-23 \mathrm{~S})$ internally transcribed spacer (ITS) sequences as a more variable genetic marker for assessing the phylogenetic relationships of the genus Nocardioides and related taxa. Recently, sequencing (Leblond-Bourget et al., 1996; Zavaleta et al., 1996; Yoon et al., 1997b), restriction analysis (Navarro et al., 1992) and molecular typing (Dolzani et al., 1994) of 16S-23S ITS regions have been shown to be useful in inferring the phylogenetic relationships between closely related organisms (Gürtler \& Stanisich, 1996). The $16 \mathrm{~S}-23 \mathrm{~S}$ ITS region can be a useful target for polyphasic taxonomic analysis (Vandamme et al., 1996). The sizes of 16S-23S ITS regions in many organisms are not conserved, unlike those of 16S rDNA/rRNA molecules (Gürtler \& Stanisich, 1996; Leblond-Bourget et al., 1996; Yoon et al., 1997b). This property may also be useful for discriminating between genera, between species and between strains of a given species.

The aim of this study was to investigate intra- and interspecific relationships of the genus Nocardioides and between two Aeromicrobium species based on 16S-23S ITS sequences. In particular, the 16S-23S ITS sequence was thought to be helpful for studying the phylogenetic relationships between closely related strains and species of the genus Nocardioides. An additional objective was to investigate whether it is possible to clarify intergeneric relationships between the genera Nocardioides and Aeromicrobium, which are in fact phylogenetically related to each other despite differences in chemotaxonomic characteristics, by sequence difference and size differentiation of $16 \mathrm{~S}-23 \mathrm{~S}$ 
ITS sequences. The ITS sequences determined in this study were also compared with those of representative members of other genera to investigate whether ITS sequences are appropriate for inferring phylogenetic relationships between distantly related organisms.

\section{METHODS}

Organisms and culture conditions. The strains used in this study are listed in Table 1. All strains used in this study were grown in shake flasks containing tryptone soy broth supplemented with glucose $(0.75 \%, \mathrm{w} / \mathrm{v})$ at temperatures appropriate for each strain. The broth cultures were checked for purity before they were harvested by centrifugation.

Isolation of chromosomal DNA. Chromosomal DNAs were isolated by the method described previously (Yoon et al., 1996).

PCR amplification and sequencing of 16S-23S ITS regions. The primers for amplification of the DNA fragment containing the 16S-23S ITS region have been described previously (Yoon et al., 1997b). The sequences of the oligonucleotide primers annealing to the $16 \mathrm{~S}$ rDNA and 23S rDNA regions were 5'-CAGCMGCCGCGGTAATSC-3' [primer 16SF, positions 519-536 (Escherichia coli 16S rRNA numbering)] and 5'-AGGCATCCACCGTGCGCCCT-3' [primer 23SR, positions 34-14 (E. coli 23S rRNA numbering)], respectively. The $5^{\prime}$ end of primer $16 \mathrm{SF}$ was phosphorylated by using T4 polynucleotide kinase (New England Biolabs). PCR was performed as described previously (Yoon et al., 1997b, c) using phosphorylated primer $16 \mathrm{SF}$ and primer 23SR. The PCR product was recovered by precipitation with 2-propanol, and the strand containing phosphorylated primer from the PCR product was selectively digested using $\lambda$ exonuclease according to the instructions included with the Strandase ssDNA preparation kit (Novagen). Sequencing of the 16S-23S ITS region was performed with the ssDNA template produced as described previously (Kim et al., 1995). The primers used to sequence the $16 \mathrm{~S}-23 \mathrm{~S}$ ITS region include several primers designed from $16 \mathrm{~S}-23 \mathrm{~S}$ ITS regions in addition to universal primers within $16 \mathrm{~S}$ rDNA, namely $1392 \mathrm{~F}$ [5'-GYACACACCGCCCGT-3', positions 1392-1406 ( $E$. coli 16S rRNA numbering)] (Lane et al., 1985) and 1525F [5'-GGCTGGATCACCTCCTTTCT-3', positions 1525-1542 (E. coli $16 \mathrm{~S}$ rRNA numbering)] (Stackebrandt \& Liesack, 1993), which have been described previously.

Cloning and sequencing of the 16S-23S ITS region from Nocardioides sp. NSP36. The PCR product of the $16 \mathrm{~S}-23 \mathrm{~S}$ ITS region for cloning was amplified by using a primer annealing to the $3^{\prime}$ end of $16 \mathrm{~S}$ rDNA, primer $1525 \mathrm{~F}$ and primer 23SR. Cloning and sequencing of the PCR product was performed using the methods described previously (Sambrook et al., 1989; Kim et al., 1995). The sequence of the $16 \mathrm{~S}-23 \mathrm{~S}$ ITS region was determined using three to four clones.

Data analysis. The sequences of the $16 \mathrm{~S}-23 \mathrm{~S}$ ITS sequences determined in this study were aligned by using CLUSTAL $w$ software (version 1.7) (Thompson et al., 1994). The 16S-23S ITS sequences of representatives strains or all strains of the genus Nocardioides, two Aeromicrobium species and Terrabacter tumescens were aligned with those of some other taxa whose 16S-23S ITS sequences have been determined. Reference sequences used in this study were obtained from the GenBank databases, where the nucleotide sequences are available under the following accession numbers: M27245 (Streptomyces ambofaciens), M76388 (Streptomyces griseus subsp. griseus), M20148 ('Streptomyces lividans'), M55343 (Frankia sp.), U09517 (Bifidobacterium bifidum) and U09520 (Bifidobacterium breve). The nucleotide similarity values were calculated from the alignment, and a tree was constructed by using the neighbour-joining method (Saitou \& Nei, 1987) from a distance matrix calculated with CLUSTAL w (Thompson et al., 1994).

\section{RESULTS AND DISCUSSION}

The DNA fragments containing the 16S-23S ITS regions from 33 strains of the genus Nocardioides, two Aeromicrobium species and Terrabacter tumescens were amplified by PCR using two primers designed from the conserved regions of $16 \mathrm{~S}$ and $23 \mathrm{~S}$ rDNAs. All of the strains produced one PCR product for the 16S-23S ITS-containing DNA fragment as determined by agarose gel electrophoresis of PCR amplicons (data not shown). The presence of one PCR product for the 16S-23S ITS has been described for some Bifidobacterium species (Leblond-Bourget et al., 1996) and Saccharomonospora species. However, the 16S-23S ITS sequences of some strains used in this study were found to differ in terms of size and sequence from $r r n$ loci, as determined by subsequent sequence analysis. Variation in the length in 16S-23S ITS regions due to heterogeneity among rDNA alleles of a given strain has been found in some organisms (Gürtler \& Stanisich, 1996; Hain et al., 1997). In particular, the 16S-23S ITS sequences of Staphylococcus aureus and Haemophilus influenzae, for which the sequence of complete sets of rRNA operons has been determined, vary from 303 to $551 \mathrm{bp}$ and from 478 to $723 \mathrm{bp}$, respectively (Gürtler \& Stanisich, 1996). The heterogeneity of size and number of 16S-23S ITS sequences of rDNA alleles has been used to discriminate between strains of some Streptomyces species (Hain et al., 1997). PCR products of heterogeneous size and sequence present in a single PCR amplicon can yield unreadable mixed sequence data when PCR products of the 16S-23S ITS region are sequenced directly.

The nucleotide sequences of the 16S-23S ITS regions have been determined by directly sequencing nonphosphorylated single strands of the PCR amplicon obtained using $\lambda$ exonuclease, which selectively digests 5 -phosphorylated strands of double-stranded DNA fragments. The $5^{\prime}$ ends of the $16 \mathrm{~S}-23 \mathrm{~S}$ ITS regions of strains used in this study were determined from the $3^{\prime}$ ends of the 16S rDNAs of Bacillus subtilis (Green et al., 1985), Frankia sp. (Normand et al., 1992) and some Streptomyces species (Baylis \& Bibb, 1988; Suzuki \& Yamada, 1988; Suzuki et al., 1988; Pernodet et al., 1989; Van Wezel et al., 1991; Kim et al., 1993). The 3' ends of the 16S-23S ITS regions were deduced by comparison with the similar sequence of the $5^{\prime}$ end regions of the $23 \mathrm{~S}$ rDNAs of Bacillus subtilis (Green et al., 1985), Frankia sp. (Normand et al., 1992) and some Streptomyces species (Baylis \& Bibb, 1988; Suzuki et 
Table 1. Strains used in this study, and sizes and accession numbers for $16 \mathrm{~S}-235$ ITS sequences

\begin{tabular}{|c|c|c|c|c|}
\hline Lab. no. & Species & Source* & Size (bp) & Accession no. \\
\hline $\mathrm{NSP} \mathrm{1}^{\mathrm{T}}$ & Nocardioides albus & КСТC $9186^{\mathrm{T}}$ & 514 & $\mathrm{AF} 017470$ \\
\hline \multirow[t]{2}{*}{ NSP02 } & Nocardioides albus & JCM 5851 & 499 & $\mathrm{AF} 017471(\mathrm{rrn} 1)$ \\
\hline & & & 500 & $\mathrm{AF} 017472(r r n 2)$ \\
\hline NSP03 & Nocardioides albus & JCM 5852 & 513 & AF017473 \\
\hline NSP04 & Nocardioides albus & JCM 5853 & 468 & AF017474 \\
\hline NSP05 & Nocardioides albus & JCM 5854 & 520 & AF017475 \\
\hline NSP06 & Nocardioides albus & JCM 5855 & 512 & AF017476 \\
\hline NSP07 & Nocardioides albus & JCM 5856 & 512 & AF017477 \\
\hline NSP08 & Nocardioides albus & JCM 5857 & 533 & AF017478 \\
\hline NSP09 & Nocardioides albus & JCM 5858 & 510 & AF017479 \\
\hline NSP10 & Nocardioides albus & DSM 46107 & 509 & AF017480 \\
\hline NSP11 & Nocardioides albus & JCM 5859 & 514 & AF017481 \\
\hline NSP12 & Nocardioides albus & JCM 5860 & 507 & AF017482 \\
\hline NSP13 & Nocardioides albus & JCM 5861 & 539 & $\mathrm{AF} 017483$ \\
\hline \multirow[t]{2}{*}{ NSP14 } & Nocardioides albus & JCM 5862 & 509 & $\mathrm{AF} 017484(r r n 1)$ \\
\hline & & & & $\mathrm{AF} 017485(\mathrm{rrn} 2)$ \\
\hline NSP15 & Nocardioides albus & JCM 5863 & 531 & AF017486 \\
\hline NSP16 & Nocardioides albus & JCM 5864 & 509 & AF017487 \\
\hline NSP17 & Nocardioides albus & DSM 43873 & 505 & AF017488 \\
\hline \multirow[t]{2}{*}{ NSP18 } & Nocardioides albus & DSM 43874 & 511 & $\mathrm{AF} 017489(\mathrm{rrn} 1)$ \\
\hline & & & 523 & $\mathrm{AF} 017490(\mathrm{rrn} 2)$ \\
\hline $\mathrm{NSP}_{19}{ }^{\mathrm{T}}$ & Nocardioides jensenii & KCTC $9134^{\mathrm{T}}$ & 344 & AF017491 \\
\hline $\mathrm{NSP} 20^{\mathrm{T}}$ & Nocardioides luteus & KCTC $9575^{\mathrm{T}}$ & 473 & $\mathrm{AF} 017492$ \\
\hline $\mathrm{NSP} 21^{\mathrm{T}}$ & Nocardioides plantarum & NCIMB $12834^{\mathrm{T}}$ & 438 & AF017493 \\
\hline $\mathrm{NSP} 22^{\mathrm{T}}$ & Nocardioides simplex & KCTC $9106^{\mathrm{T}}$ & 386 & AF017494 \\
\hline NSP23 & Nocardioides simplex & JCM 1366 & 386 & AF017495 \\
\hline NSP24 & Nocardioides simplex & JCM 1367 & 386 & AF017496 \\
\hline \multirow[t]{2}{*}{ NSP25 } & Nocardioides simplex & NCIMB 12919 & 387 & $\mathrm{AF} 017497(r r n 1)$ \\
\hline & & & 388 & $\mathrm{AF} 017498(r r n$ 2) \\
\hline NSP27 & Nocardioides simplex & ATCC 15799 & 386 & AF017499 \\
\hline NSP30 ${ }^{\mathrm{T}}$ & 'Nocardioides flavus' & IFO $14396^{\mathrm{T}}$ & 473 & AF017500 \\
\hline NSP31 & 'Nocardioides flavus' & IFO 14397 & 473 & AF017501 \\
\hline $\mathrm{NSP} 2^{\mathrm{T}}$ & 'Nocardioides fulvus' & $\mathrm{JCM} 3335^{\mathrm{T}}$ & 473 & AF017502 \\
\hline NSP33 & 'Nocardioides fulvus' & IFO 14399 & 473 & AF017503 \\
\hline \multirow[t]{2}{*}{ NSP36 } & Nocardioides sp. & ATCC 39419 & 429 & AF017504 (rrn 1) \\
\hline & & & 439 & $\mathrm{AF} 017505(r r n 2)$ \\
\hline NSP40 & 'Nocardioides pyridinolyticus' & OS4, S.-K. Rhee & 392 & $\mathrm{AF} 017506$ \\
\hline NSP41 & Nocardioides-like isolate & Y. K. Cho & 328 & AF017507 \\
\hline NSP37 $7^{T}$ & Aeromicrobium erythreum & NRRL B-3381 ${ }^{\mathrm{T}}$ & 349 & AF017508 \\
\hline NSP38 ${ }^{\mathrm{T}}$ & Aeromicrobium fastidiosum & КСТС $9576^{\mathrm{T}}$ & 355 & AF017509 \\
\hline NSP39 $9^{T}$ & Terrabacter tumescens & КСТC $9133^{T}$ & 386 & AF017510 \\
\hline
\end{tabular}

* KCTC, Korean Collection for Type Cultures, Taejon, Korea; JCM, Japan Collection of Microorganisms, Institute of Physical and Chemical Research, Saitama, Japan; DSM, Deutsche Sammlung von Mikroorganismen und Zellkulturen, Braunschweig, Germany; NCIMB, National Collection of Industrial and Marine Bacteria, NCIMB, Aberdeen, UK; ATCC, American Type Culture Collection, Rockville, MD, USA; IFO, Institute for Fermentation, Osaka, Japan; NRRL, Agricultural Research Service Culture Collection, National Center for Agricultural Utilization Research, Peoria, IL, USA.

al., 1988; Pernodet et al., 1989; Kim et al., 1993). The lengths of the 16S-23S ITS sequences of the Nocardioides strains determined ranged from $328 \mathrm{bp}$ in Nocardioides-like isolate NSP41 to 539 bp in N. albus NSP13 (Table 1). Large size differences between
16S-23S ITS sequences from different strains have also been found in some Bifidobacterium species (LeblondBourget et al., 1996), with 16S-23S ITS sequences from some strains being only half the size of those found in other strains belonging to the same species. 
The size variation of $16 \mathrm{~S}-23 \mathrm{~S}$ ITS sequences, together with sequence divergence, can be useful in discriminating between strains belonging to the same species and between species belonging to the same genus, especially in the case of closely related organisms. The lengths of the $16 \mathrm{~S}-23 \mathrm{~S}$ ITS sequences of $N$. simplex strains showed a small intraspecific variation of 386388 bp when compared with $N$. albus strains (Table 1). The 16S-23S ITS regions of $N$. jensenii $\mathrm{NSP}^{\mathrm{T}} \mathrm{T}^{\mathrm{T}}, N$. luteus $\mathrm{NSP} 20^{\mathrm{T}}$ and $N$. plantarum NSP $21^{\mathrm{T}}$ were 344 , 473 and $438 \mathrm{bp}$, respectively (Table 1). The 16S-23S ITS sequences of ' $N$. flavus' strains NSP30 ${ }^{\mathrm{T}}$ and NSP31 and ' $N$. fulvus' NSP032 ${ }^{\mathrm{T}}$ were the same size as those of $N$. luteus NSP20T (Table 1). $N$. albus NSP05, $N$. luteus $\mathrm{NSP} 20^{\mathrm{T}}$, ' $N$. flavus' strains NSP30 ${ }^{\mathrm{T}}$ and NSP31 and ' $N$. fulvus' NSP032 ${ }^{\mathrm{T}}$ were shown to have the same $16 \mathrm{~S}$ rDNA sequence according to our recent 16S rDNA sequence analysis (Yoon et al., 1998). However, the 16S-23S ITS of $N$. albus NSP05 was 47 bp longer. The 16S-23S ITS sequences of $A$. erythreum NSP $37^{\mathrm{T}}$ and $A$. fastidiosum NSP $38^{\mathrm{T}}$ were 349 and 355 bp long, respectively, which is smaller than those of Nocardioides strains except $N$. jensenii NSP19 ${ }^{\mathrm{T}}$ and Nocardioides sp. NSP41 (Table 1). The length of the 16S-23S ITS region of $T$. tumescens NSP39 ${ }^{\mathrm{T}}$ was 386 bp (Table 1).

Sequence analysis revealed that some Nocardioides strains used in this study displayed heterogeneity in the 16S-23S ITS sequences between rRNA gene clusters. From direct sequencing of the 16S-23S ITS region, unreadable mixed sequence data were produced above position 237 of the 16S-23S ITS sequence for N. albus NSP02, above position 124 for $N$. albus NSP18, between positions 169 and 173 and above position 183 for $N$. simplex NSP25 and above position 15 for Nocardioides sp. NSP36. However, N. albus NSP14 yielded unreadable mixed sequence data only between positions 373 and 393 of the 16S-23S ITS sequence, which indicates that the region exhibits sequence divergence but not size divergence between rDNA alleles. When we performed sequence analysis of several clones containing $16 \mathrm{~S}-23 \mathrm{~S}$ ITS sequences to investigate the extent of heterogeneity of $16 \mathrm{~S}-23 \mathrm{~S}$ ITS sequences from the five strains, all five strains were found to have two types of $16 \mathrm{~S}-23 \mathrm{~S}$ ITS sequences. In our previous ribotyping study, all of the Nocardioides strains and two Aeromicrobium species used in this study were shown to have two copies of rDNA alleles on their genomes (data not shown). Thus, N. albus strains NSP02, NSP14 and NSP18, N. simplex NSP25 and Nocardioides sp. NSP36 were thought to have two types of 16S-23S ITS sequences derived from the two rRNA gene clusters. However, in our study, the sequence and size divergence of two rRNA gene clusters of the five Nocardioides strains were not investigated in two $r$ rn loci distinguished by the use of restriction endonucleases. The rRNA gene cluster containing the smaller 16S-23S ITS and the rRNA gene cluster containing the larger 16S-23S ITS from $N$. albus strains NSP02 and NSP18, N. simplex NSP25 and Nocardioides sp. NSP36 were designated $r r n 1$ and $r r n$ 2, respectively. In $N$. albus NSP14, the rRNA gene cluster with a 16S-23S ITS containing the nucleotide sequence GGCTGTATGGGTTTACCTGTC and the rRNA gene cluster with a 16S-23S ITS containing the nucleotide sequence of AGCCGTGGGTTTAGGCCTGGT between positions 373 and 393 were designated $r r n 1$ and $r r n 2$, respectively. N. albus NSP02 was found to have $16 \mathrm{~S}-23 \mathrm{~S}$ ITS sequences that were 499 bp long ( $r r n 1$ ) and $500 \mathrm{bp}$ long ( $r r n$ 2), and the 16S-23S ITS sequence of $r r n 2$ was identical to that of $r r n 1$ except that it contained an additional $G$ nucleotide between positions 236 and 237 of the rrn $116 \mathrm{~S}-23 \mathrm{~S}$ ITS. $N$. albus NSP18 had two types of 16S-23S ITS sequences which differed in size by $12 \mathrm{bp}$ (Table 1). The larger 16S-23S ITS of $N$. albus NSP18 ( $r r n$ 2) differed in size and sequence from the smaller one ( $r r n 1)$ between positions 124 and 129 , resulting in a nucleotide similarity level of $99.6 \%$ and a nucleotide similarity level, with gaps included, of $97 \cdot 3 \%$. N. simplex NSP25 had two types of $16 \mathrm{~S}-23 \mathrm{~S}$ ITS, which show sequence divergence between positions 169 and 173, and the larger type ( $r r n$ 2) of 16S-23S ITS contains an additional A nucleotide between positions 183 and 184 of the smaller type ( $r r n$ 1). The nucleotide similarity and the nucleotide similarity level with gaps included between two types of $16 \mathrm{~S}-23 \mathrm{~S}$ ITS sequences of $N$. simplex NSP25 are 98.4 and $98.2 \%$, respectively. It was interesting that the two types of 16S-23S ITS sequences found in Nocardioides sp. NSP36 differed in size by $10 \mathrm{bp}$ and showed high sequence divergence, with the nucleotide similarity level being $80.8 \%$ (Table 2 ) and $78.4 \%$ with gaps included.

All strains were analysed to investigate the existence of tRNA-like structures within their 16S-23S ITS regions. Comparisons of $16 \mathrm{~S}-23 \mathrm{~S}$ ITS sequences determined in this study with 21 tRNA sequences published previously (Green \& Vold, 1983) showed no similarities. Even sequences within 16S-23S ITS sequences of certain strains showing few matches with tRNA sequences did not form the secondary structure characteristic of tRNA. Moreover, the sequences did not contain the trinucleotide sequence (-CCA) that has been found at the $3^{\prime}$ end of most tRNA sequences. Thus, it was concluded that no strain used in this study contains tRNA sequences in intergenic spacers between $16 \mathrm{~S}$ and $23 \mathrm{~S}$ rDNAs, as shown previously for some Streptomyces species (Baylis \& Bibb, 1988; Suzuki et al., 1988; Pernodet et al., 1989; Kim et al., 1993), Frankia strain (Normand et al., 1992), some Bifidobacterium species (Leblond-Bourget et al., 1992) and Saccharomonospora strains (Yoon et al., 1997b). Our results are consistent with the previous finding that there are no tRNA genes in the 16S-23S ITS sequences of members of the order Actinomycetales.

The strains of $N$. albus showed a high degree of sequence divergence among their 16S-23S ITS sequences. $N$. albus strains NSP01 $1^{\mathrm{T}}$ and NSP11 and $N$. albus strains NSP06 and NSP07 were found to have identical 16S-23S ITS sequences. The mean intra- 
Table 2. Percentage nucleotide similarity for $16 \mathrm{~S}-23 \mathrm{~S}$ ITS sequences of representatives of the genus Nocardioides, two Aeromicrobium species, Terrabacter tumescens and representatives of some other taxa

\begin{tabular}{|c|c|c|c|c|c|c|c|c|c|c|c|c|c|c|c|c|c|c|c|c|}
\hline & 1 & 2 & 3 & 4 & 5 & 6 & 7 & 8 & 9 & 10 & 11 & 12 & 13 & 14 & 15 & 16 & 17 & 18 & 19 & 20 \\
\hline \multicolumn{21}{|l|}{ 1. N. albus $\mathrm{NSP} 1^{\mathrm{T}}$} \\
\hline 2. N. jensenii $\mathrm{NSP} 19^{\mathrm{T}}$ & 65.8 & & & & & & & & & & & & & & & & & & & \\
\hline 3. N. luteus NSP20 ${ }^{\mathrm{T}}$ & 84.8 & $63 \cdot 3$ & . & & & & & & & & & & & & & & & & & \\
\hline 4. $N$. plantarum $\mathrm{NSP} 21^{\mathrm{T}}$ & $64 \cdot 9$ & 53.8 & $59 \cdot 6$ & & & & & & & & & & & & & & & & & \\
\hline 5. N. simplex NSP22 $2^{\mathrm{T}}$ & $55 \cdot 1$ & $51 \cdot 3$ & 57.9 & $66 \cdot 7$ & & & & & & & & & & & & & & & & \\
\hline $6 .^{\prime} N$. flavus' $^{\prime} \mathrm{NSP} 30^{\mathrm{T}}$ & $85 \cdot 0$ & $63 \cdot 0$ & 99.8 & $59 \cdot 8$ & 58.6 & & & & & & & & & & & & & & & \\
\hline $7 .{ }^{\prime} N$. fulvus' NSP $32^{\mathrm{T}}$ & $84 \cdot 8$ & $63 \cdot 3$ & 100 & $59 \cdot 6$ & $58 \cdot 1$ & $99 \cdot 8$ & & & & & & & & & & & & & & \\
\hline $8 .{ }^{\prime}$ N. fulvus' NSP33 & $63 \cdot 6$ & $68 \cdot 0$ & $54 \cdot 8$ & $56 \cdot 6$ & $55 \cdot 3$ & $54 \cdot 4$ & $54 \cdot 8$ & & & & & & & & & & & & & \\
\hline 9. Nocardioides sp. NSP36 (rm 1) & 57.6 & $70 \cdot 2$ & $55 \cdot 3$ & $58 \cdot 1$ & $56 \cdot 1$ & $55 \cdot 6$ & $55 \cdot 3$ & $75 \cdot 4$ & & & & & & & & & & & & \\
\hline 10. Nocardioides sp. NSP36 ( $\mathrm{rm} 2)$ & $54 \cdot 8$ & $71 \cdot 5$ & $49 \cdot 2$ & $53 \cdot 1$ & 56.6 & $49 \cdot 4$ & $49 \cdot 2$ & 73.6 & 80.8 & & & & & & & & & & & \\
\hline 11. Nocardioides sp. NSP40 & 63.9 & $64 \cdot 2$ & $66 \cdot 8$ & $65 \cdot 3$ & $54 \cdot 4$ & $66 \cdot 2$ & $66 \cdot 8$ & 58.4 & $58 \cdot 4$ & $57 \cdot 7$ & & & & & & & & & & \\
\hline 12. Nocardioides sp. NSP41 & 53.6 & $48 \cdot 4$ & 540 & 71.5 & $82 \cdot 8$ & $53 \cdot 2$ & $54 \cdot 0$ & $61 \cdot 0$ & $70 \cdot 4$ & $59 \cdot 0$ & 57.6 & & & & & & & & & \\
\hline 13. A, erythreum NSP37 $7^{\mathrm{T}}$ & $66 \cdot 8$ & $73 \cdot 1$ & $67 \cdot 4$ & $57 \cdot 4$ & $52 \cdot 9$ & $67 \cdot 7$ & $67 \cdot 4$ & $55 \cdot 4$ & $64 \cdot 9$ & $62 \cdot 5$ & $66 \cdot 4$ & $52 \cdot 7$ & & & & & & & & \\
\hline 14. A. fastidiosum NSP38 & 56.5 & $70 \cdot 1$ & $54 \cdot 0$ & $54 \cdot 5$ & $56 \cdot 8$ & $58 \cdot 4$ & $54 \cdot 0$ & 68.6 & $65 \cdot 4$ & 64.6 & 57.0 & $49 \cdot 5$ & $71 \cdot 2$ & & & & & & & \\
\hline 15. T. tumescens $\mathrm{NSP} 39^{\mathrm{T}}$ & 62.5 & $66 \cdot 3$ & $57 \cdot 1$ & $60 \cdot 1$ & $52 \cdot 8$ & $57 \cdot 4$ & $57 \cdot 1$ & $66 \cdot 9$ & $61 \cdot 6$ & $60 \cdot 6$ & $59 \cdot 4$ & $55 \cdot 1$ & $68 \cdot 7$ & $65 \cdot 3$ & & & & & & \\
\hline 16. S. ambofaciens & 57.6 & 66.6 & $62 \cdot 7$ & $58 \cdot 3$ & $54 \cdot 4$ & $61 \cdot 1$ & $62 \cdot 7$ & $65 \cdot 9$ & $72 \cdot 6$ & $75 \cdot 8$ & $62 \cdot 7$ & $53 \cdot 0$ & $65 \cdot 3$ & $65 \cdot 4$ & 67.9 & & & & & \\
\hline 17. S. griseus subsp. griseus & $64 \cdot 7$ & $66 \cdot 4$ & $64 \cdot 7$ & $58 \cdot 1$ & $52 \cdot 4$ & $65 \cdot 1$ & $64 \cdot 7$ & $58 \cdot 2$ & 67.8 & $66 \cdot 6$ & 56.6 & 50.9 & $62 \cdot 9$ & $65 \cdot 3$ & 66.8 & 76.7 & & & & \\
\hline 18.' S. lividans' & $67 \cdot 4$ & $69 \cdot 9$ & $67 \cdot 8$ & $56 \cdot 5$ & 67.2 & $68 \cdot 5$ & $67 \cdot 8$ & $61 \cdot 8$ & $66 \cdot 2$ & $73 \cdot 5$ & 67.5 & $46 \cdot 9$ & $70 \cdot 0$ & 67.2 & $70 \cdot 0$ & $81 \cdot 0$ & $77 \cdot 9$ & & & \\
\hline 19. Frankia strain ORS 020606 & $58 \cdot 0$ & 67.2 & $54 \cdot 1$ & $62 \cdot 4$ & 52.5 & $54 \cdot 3$ & $54 \cdot 1$ & $65 \cdot 6$ & $62 \cdot 8$ & $61 \cdot 4$ & 48.7 & $53 \cdot 9$ & $68 \cdot 8$ & $65 \cdot 6$ & $66 \cdot 4$ & $71 \cdot 3$ & $68 \cdot 4$ & $72 \cdot 5$ & & \\
\hline 20. B. bifidum & $55 \cdot 6$ & $49 \cdot 7$ & $59 \cdot 7$ & 57.6 & $61 \cdot 5$ & $59 \cdot 4$ & $59 \cdot 7$ & $50 \cdot 4$ & $53 \cdot 4$ & $51 \cdot 4$ & 51.5 & $65 \cdot 6$ & $51 \cdot 7$ & $53 \cdot 7$ & 53.9 & $56 \cdot 8$ & $54 \cdot 6$ & $58 \cdot 4$ & $54 \cdot 3$ & \\
\hline 21. B. breve & $54 \cdot 6$ & $56 \cdot 0$ & $51 \cdot 2$ & $54 \cdot 1$ & 58.8 & $51 \cdot 7$ & $51 \cdot 2$ & 50.6 & $50 \cdot 6$ & $50 \cdot 1$ & $58 \cdot 0$ & $57 \cdot 1$ & $52 \cdot 3$ & $56 \cdot 3$ & $58 \cdot 2$ & $55 \cdot 8$ & 57.0 & $55 \cdot 1$ & 56.6 & $70 \cdot 6$ \\
\hline
\end{tabular}

specific nucleotide similarity value for $16 \mathrm{~S}-23 \mathrm{~S}$ ITS sequences of $N$. albus was $92 \cdot 1 \pm 7 \cdot 9 \%$. The most distant relationship was that between $N$. albus NSP04 and $N$. albus strains NSP06 and NSP07, for which a level of similarity of $84 \cdot 1 \%$ was obtained. The $16 \mathrm{~S}-23 \mathrm{~S}$ ITS sequence has been found to have a high frequency of insertion and deletion, resulting in a high rate of evolution (Gürtler \& Stanisich, 1996; LeblondBourget et al., 1996; Yoon et al., 1997b). The number of insertions and deletions in alignments of $16 \mathrm{~S}-23 \mathrm{~S}$ ITS sequences can constitute important differences between organisms. Thus, nucleotide similarities including gap sites for each strain were also calculated in our study. The mean level of nucleotide similarity including gap sites for $N$. albus strains was $87 \cdot 3 \pm 12 \cdot 7 \%$. The most distant relationship, including gaps, was the relationship between $N$. albus NSP05 and $N$. albus NSP09, which exhibited a level of nucleotide similarity of $74.5 \%$. Including gap sites in the calculation of the nucleotide similarity values helps clarify the relationships among $N$. albus strains. The high degree of sequence divergence of 16S-23S ITS sequences among $N$. albus strains has been shown to be useful in discriminating $N$. albus strains. Also, despite their sequence divergences, $N$. albus strains form phylogenetic lineages that are distinct from other Nocardioides species, except $N$. luteus $\mathrm{NSP}^{\mathrm{T}} 0^{\mathrm{T}}$, $N$. flavus' strains NSP30 ${ }^{\mathrm{T}}$ and NSP31 and ' $N$. fulvus' NSP $32^{\mathrm{T}}$, and from members of the other genera in the phylogenetic tree (data not shown). The 16S-23S ITS sequences of $N$. luteus $\mathrm{NSP} 20^{\mathrm{T}}$ are $85.0-93.0 \%$ similar to those of $N$. albus strains, and $76 \cdot 0-88.6 \%$ similar to $N$. albus strains when gaps are included. When the $16 \mathrm{~S}-23 \mathrm{~S}$ ITS of $N$. luteus NSP20 $0^{\mathrm{T}}$ was compared with that of $N$. albus NSP05 (JCM 5854), whose 16S rDNA sequence has been shown to be identical to that of $N$. luteus NSP20 ${ }^{\mathrm{T}}$ (Yoon et al., 1998), the level of nucleotide similarity and of nucleotide similarity in- cluding gaps was 90.0 and $77.0 \%$, respectively. The $16 \mathrm{~S}-23 \mathrm{~S}$ ITS of $N$. luteus NSP $20^{\mathrm{T}}$ was only $1 \mathrm{bp}$ different from those of ' $N$. flavus' strains NSP $30^{\mathrm{T}}$ and NSP31, whose nucleotide sequences are identical, and had an identical sequence to that of ' $N$. fulvus' NSP32T ' ' $N$. flavus' strains NSP30 ${ }^{\mathrm{T}}$ and NSP31 and ' $N$. fulvus' NSP $32^{\mathrm{T}}$ are thought to be closely related to $N$. luteus NSP $20^{\mathrm{T}}$ from the results of the $16 \mathrm{~S}-23 \mathrm{~S}$ ITS sequence analysis and from that fact that the $16 \mathrm{~S}$ rDNA similarity value is $100 \%$, as found previously. Organisms with an identical sequence or only a $1 \mathrm{bp}$ sequence difference in the $16 \mathrm{~S}-23 \mathrm{~S}$ ITS sequences, although showing high sequence divergence from other Nocardioides species, should be regarded as members of same species. Thus, it is proposed that ' $N$. flavus' strains NSP $30^{\mathrm{T}}$ and NSP31 and ' $N$. fulvus' NSP $32^{\mathrm{T}}$ be described as members of $N$. luteus. ' $N$. flavus' NSP $30^{\mathrm{T}}$ has been assumed to be a synonym of $N$. luteus (Prauser, 1989). N. luteus NSP20 $0^{\mathrm{T}}$ clearly showed a lower 16S-23S ITS similarity to $N$. albus strains, when compared with the results of 16S rDNA analysis. However, $N$. albus strains and $N$. luteus $\mathrm{NSP} 20^{\mathrm{T}}$ form a combined phylogenetic group in a $16 \mathrm{~S}-23 \mathrm{~S}$ ITS sequence-based tree (data not shown). The degree of 16S-23S ITS similarity $(85.0-93.0 \%)$ between $N$. luteus NSP20 $0^{\mathrm{T}}$ and $N$. albus strains falls within in the range of $16 \mathrm{~S}-23 \mathrm{~S}$ ITS similarity found in $N$. albus strains $(84 \cdot 1-100 \%)$. In addition, the $16 \mathrm{~S}-23 \mathrm{~S}$ ITS sequences of $N$. luteus NSP $20^{\mathrm{T}}$ are $473 \mathrm{bp}$, which falls within the range of lengths of 16S-23S ITS sequences found in $N$. albus strains (468-539 bp). However, the nucleotide similarity level between $N$. luteus NSP $20^{\mathrm{T}}$ and $N$. jensenii $\mathrm{NSP}^{2} 9^{\mathrm{T}}, N$. plantarum $\mathrm{NSP} 21^{\mathrm{T}}$ and $N$. simplex NSP $22^{\mathrm{T}}$ was $63.3,59.6$ and $57.9 \%$, respectively. The sequence divergences of the 16S-23S ITS between $N$. luteus NSP $20^{\mathrm{T}}$ and $N$. albus strains show clearer results than those of $16 \mathrm{~S}$ rDNAs, which have nucleotide similarity of $99.5 \pm 0 \cdot 5 \%$. Nevertheless, the taxonomic 
relationship between the two taxa has been vague until now. Re-examination of DNA-DNA relatedness is thought to be the ultimate solution to elucidating the relationships between $N$. albus strains, $N$. luteus NSP20 $0^{\mathrm{T}}$, ' $N$. flavus' strains NSP30 $0^{\mathrm{T}}$ and NSP31 and ' $N$. fulvus' NSP32

' $N$. fulvus' NSP33 showed a low 16S-23S ITS similarity to ' $N$. fulvus' NSP $32^{\mathrm{T}}-54 \cdot 8 \%$ (Table 2 ). In the previous 16S rDNA sequence analysis, ' $N$. fulvus' NSP33 also showed low nucleotide similarity to Nocardioides strains, including ' $N$. fulvus' NSP32 ${ }^{\mathrm{T}}$, and Aeromicrobium species, which are phylogenetically related to the genus Nocardioides, and its highest nucleotide similarity, $99 \%$, was with Nocardioides $\mathrm{sp}$. NSP36 (Yoon et al., 1998). Thus, ' N. fulvus' NSP33 and Nocardioides sp. NSP36 have been considered to be members of a new genus based on phylogenetic inference and subsequently determined chemosystematic characteristics of the two strains. In this study, the 16S-23S ITS of ' $N$. fulvus' NSP33 was found to exhibit a higher degree of similarity to the 16S-23S ITS sequences of Nocardioides sp. NSP36 $(75.4 \%, r r n 1 ; 73.6 \%, r r n 2)$ than to those of other Nocardioides strains and Aeromicrobium species (Table 2). The degree of similarity between 16S-23S ITS sequences from ' $N$. fulvus' NSP33 and Nocardioides sp. NSP36 and those of other Nocardioides strains and Aeromicrobium species are similar those between Nocardioides species and between Nocardioides species and Aeromicrobium species.

The levels of nucleotide similarity of $16 \mathrm{~S}-23 \mathrm{~S}$ ITS sequences between $N$. simplex strains varied from $97 \cdot 7$ to $100 \%$. The sizes of the $16 \mathrm{~S}-23 \mathrm{~S}$ ITS sequences were identical (386 bp) in all $N$. simplex strains except $N$. simplex NSP25, which has two types of 16S-23S ITS sequences -387 and 388 bp. $N$. simplex strains NSP $22^{\mathrm{T}}$ and NSP27 shared an identical 16S-23S ITS sequence, and $N$. simplex strains NSP23 and NSP24 also shared an identical 16S-23S ITS sequence. It was interesting that the nucleotide similarity value $(98.7 \%)$ between the 16S-23S ITS sequences of $N$. simplex strains NSP23 and NSP24 and the 16S-23S ITS of $r r n$ of $N$. simplex NSP25 was higher than that $(98.4 \%)$ between the two types of $16 \mathrm{~S}-23 \mathrm{~S}$ ITS sequences found in $N$. simplex NSP25. The sequence divergences of $16 \mathrm{~S}-23 \mathrm{~S}$ ITS sequences found between $N$. simplex strains were much smaller than those found between $N$. albus strains. Previous additional $N$. simplex strains, namely $N$. simplex strains ATCC 13260, ATCC 19565 and ATCC 19566, were reclassified as Rhodococcus erythropolis Gray and Thornton (Gray \& Thornton, 1928) from phylogenetic inference based on $16 \mathrm{~S}$ rDNA sequences and chemotaxonomic characteristics (Goodfellow, 1989; Yoon et al., 1997d). Consequently, they were not used in this study. Nocardioides-like isolate NSP41, whose $16 \mathrm{~S}$ rDNA is $98.6 \%$ similar to that of N. simplex strains (Yoon et al., 1998), was also analysed with respect to the $16 \mathrm{~S}-23 \mathrm{~S}$ ITS region to investigate the taxonomic relationships with $N$. simplex strains. Additionally, N. simplex strains NSP $22^{\mathrm{T}}$,
NSP23, NSP24, NSP25 and NSP27 were shown to have identical 16S rDNA sequences (Yoon et al., 1998). Strain NSP41 has a 16S-23S ITS of $328 \mathrm{bp}$, which is much smaller than those of $N$. simplex strains, and was $81.8-84.6 \%$ similar to that of $N$. simplex strains $(68 \cdot 4-70 \cdot 1 \%$ with gaps included). The divergence of the 16S-23S ITS of strain NSP41 from those of $N$. simplex strains show that this strain should be studied in detail by taxonomic comparison with $N$. simplex strains.

The 16S-23S ITS sequences in the type strains of validly described Nocardioides species were highly divergent, with mean nucleotide similarity values of $68 \cdot 1 \% \pm 16.8 \%$ and $59.0 \% \pm 7.7 \%$, except in relation to $N$. albus $\mathrm{NSP}^{\mathrm{T}} 1^{\mathrm{T}}$ and $\bar{N}$. luteus NSP20 ${ }^{\mathrm{T}}$. These nucleotide similarity values were sufficiently divergent to justify evaluation of the phylogenetic relationships between Nocardioides species even if nucleotide similarity values including gap sites were not calculated separately (Table 2). Moreover, $16 \mathrm{~S}-23 \mathrm{~S}$ ITS similarity values, gaps included, were too low to allow meaningful comparison between species. The most distant relationship was the relationship between $N$. jensenii NSP $19^{\mathrm{T}}$ and $N$. simplex NSP22 $2^{\mathrm{T}}$, for which a nucleotide similarity level of $51 \cdot 3 \%$ was obtained (Table 2). $N$. jensenii NSP19 $19^{\mathrm{T}}$ showed a higher $16 \mathrm{~S}-23 \mathrm{~S}$ ITS similarity to Aeromicrobium species, $T$. tumescens, ' $N$. fulvus' NSP33 and Nocardioides sp. NSP36, which have been considered to be members of another new genus, than to other Nocardioides strains (Table 2). Strain NSP40, which had been identified previously as Pimelobacter sp. (Lee et al., 1994) but has recently been described as a new species of the genus Nocardioides, 'Nocardioides pyridinolyticus' (Yoon et al., 1997a), showed a $16 \mathrm{~S}-23 \mathrm{~S}$ ITS similarity level of $54.4 \%(N$. simplex NSP22 ${ }^{\mathrm{T}}$ ) to $66.8 \%$ ( $N$. luteus NSP20 ${ }^{\mathrm{T}}$ ) to the type strains of validly described Nocardioides species. The 16S-23S ITS similarity value between Aeromicrobium erythreum NSP37 ${ }^{\mathrm{T}}$ and Aeromicrobium fastidiosum NSP $38^{\mathrm{T}}$ was $71.2 \%$ (Table 2). The two Aeromicrobium species exhibited a mutually high level of $16 \mathrm{~S}$ rRNA similarity of $98.2 \%$, despite low DNADNA relatedness values $(12-14 \%)$ between the two species (Tamura \& Yokota, 1994). Thus, the relationship between the two Aeromicrobium species was clarified by the comparison of $16 \mathrm{~S}-23 \mathrm{~S}$ ITS sequences. It is interesting that $A$. erythreum has a higher $16 \mathrm{~S}-23 \mathrm{~S}$ ITS similarity level to $N$. jensenii NSP $19^{\mathrm{T}}$ at $73 \cdot 1 \%$ than to $A$. fastidiosum. $N$. jensenii NSP $19^{T}$ was found to be more closely related to Aeromicrobium species, and even to $T$. tumescens, Frankia strain and Streptomyces species, than to Nocardioides strains from the sequence analyses of $16 \mathrm{~S}-23 \mathrm{~S}$ ITS sequences. $N$. jensenii NSP $19^{\mathrm{T}}$ was thought to be phylogenetically in the middle of the genera Nocardioides and Aeromicrobium from 16S rDNA sequence analysis, despite the fact that it has chemotaxonomic properties characteristic of the genus Nocardioides.

The 16S-23S ITS sequences of the representative strains of the genus Nocardioides, Aeromicrobium 
species and $T$. tumescens determined in this study, together with some representative species of the genera Streptomyces and Bifidobacterium and the Frankia strain, were analysed to investigate whether $16 \mathrm{~S}-23 \mathrm{~S}$ ITS sequences are also appropriate for inferring the relationships between distantly related organisms, such as intergeneric relationships. The levels of $16 \mathrm{~S}$ 23S ITS similarity between Nocardioides species, except that between $N$. albus strains and $N$. luteus NSP20 ${ }^{\mathrm{T}}$, are similar to the levels of 16S-23S ITS similarity between Nocardioides species and members of other genera used for sequence analysis in this study (Table 2). Moreover, in some instances, some Nocardioides species showed a lower 16S-23S ITS similarity level when compared with certain species of the genus Nocardioides than when compared with members of other genera (Table 2). For example, $N$. jensenii NSP19 ${ }^{\mathrm{T}}$ showed a lower similarity level to Nocardioides species $(51 \cdot 3-65.8 \%)$ than to members of the genera Aeromicrobium (70.1-73.1\%) and Streptomyces $(66.4-69.9 \%)$, T. tumescens $(66.3 \%)$ and the Frankia strain $(67.2 \%)$ (Table 2). $N$. luteus NSP20 also showed a 16S-23S ITS similarity level of $57.9 \%$ to $N$. simplex NSP $22^{\mathrm{T}}$ but showed similarity of $62 \cdot 7$ $67.8 \%$ to three species of the genus Streptomyces used in this study (Table 2). Additional inconsistent cases can be found in Table 2. There are also instances of Aeromicrobium species showing higher similarity to Streptomyces species and the Frankia strain than to certain species of the genus Nocardioides, which are members of the same family (Stackebrandt et al., 1997) (Table 2). For example, A. erythreum NSP37 ${ }^{\mathrm{T}}$ showed nucleotide similarity values of 57.4 and $52.9 \%$ to $N$. plantarum NSP $21^{\mathrm{T}}$ and $N$. simplex NSP22 $2^{\mathrm{T}}$, respectively, but showed nucleotide similarities of $68.7 \%$ to T. tumescens, $62 \cdot 9-70 \cdot 0 \%$ to Streptomyces species and $68.8 \%$ to the Frankia strain (Table 2). A. fastidiosum NSP $38^{\mathrm{T}}$ displayed nucleotide similarity values of $54.0-56.8 \%$ to the type strains of validly described Nocardioides species, except $N$. jensenii NSP19 ${ }^{\mathrm{T}}$, but showed nucleotide similarities of $65.3 \%$ to $T$. tumescens, $65 \cdot 3-67 \cdot 2 \%$ to Streptomyces species and $65.6 \%$ to the Frankia strain (Table 2). In the dendrogram based on 16S-23S ITS sequences, the genus Nocardioides, the genus Aeromicrobium, $T$. tumescens and ' $N$. fulvus' NSP33 and Nocardioides sp. NSP36, which have been considered to be members of a new genus, did not form distinct phylogenetic lineages separate from each other or from other genera used in this study. Therefore, the dendrogram is not shown.

From the above results, 16S-23S ITS sequences were not thought to be appropriate for inferring phylogenetic relationships between distantly related organisms and between high-ranked taxa. These observations are caused by large sequence variations of the 16S-23S ITS, which does not allow us to determine the relationships among distantly related organisms, such as among the genus or among the family. The large variations in 16S-23S ITS sizes, resulting from a high frequency of insertions and deletions, and the lack of nucleotide similarity make accurate alignments of 16S-23S ITS sequences difficult and, in the long run, cause unfavourable phylogenetic inferences to be made among organisms, in particular among distantly related organisms. The existence of heterogeneous 16S23S ITS sequences within a strain, which can occur as a result of lack of conservation, also makes it complicated to compare 16S-23S ITS sequences among organisms. These properties reduce the value of $16 \mathrm{~S}-$ 23S ITS sequences for database and BLAST search purposes. Nevertheless, 16S-23S ITS sequences have been shown to be reliable for inferring phylogenetic relationships among closely related organisms in this study and in previous studies (Zavaleta et al., 1996; Yoon et al., 1997b). In addition, sequence determination of the 16S-23S ITS region is relatively easy on account of its small length, irrespective of whether direct sequencing or sequencing following cloning is used, when compared with sequencing of 16S rDNA.

\section{Concluding remarks}

In conclusion, the relationships between $N$. albus strains, among $N$. albus strains, $N$. luteus NSP $20^{\mathrm{T}}$, ' $N$. flavus' strains and ' $N$. fulvus' NSP32 $2^{\mathrm{T}}$, between $N$. simplex strains, between Nocardioides-like NSP41 and $N$. simplex strains and between Aeromicrobium species, based on 16S-23S ITS sequences, are more distinct than the results obtained when $16 \mathrm{~S}$ rDNA sequences were used. The properties of $16 \mathrm{~S}-23 \mathrm{~S}$ ITS sequences also make relationships between validly described Nocardioides species, except that between $N$. albus and $N$. luteus, clearer. Nevertheless, the large sequence variabilities and length polymorphisms of 16S-23S ITS sequences make it difficult to infer relationships between the genera Nocardioides and Aeromicrobium and $T$. tumescens, and among the three taxa and other genera used in this study. It is thought that $16 \mathrm{~S}-23 \mathrm{~S}$ ITS sequences must not be used to elucidate phylogenetic relationships between high-ranked taxa or distantly related taxa such as between genera or between families.

\section{ACKNOWLEDGEMENTS}

This work was supported by grants HS1331 from the Ministry of Science and Technology of the Republic of Korea. We are grateful to Martin Hector for aiding data analysis, to Russell Davidson for reviewing the manuscript, and to J. L. Swezey of the ARS Patent Culture Collection, Microbial Properties Research for providing Aeromicrobium erythreum NRRL B-3381.

\section{REFERENCES}

Baylis, H. A. \& Bibb, M. J. (1988). Transcriptional analysis of the 16S rRNA gene of the rrnD gene set of Streptomyces coelicolor A3(2). Mol Microbiol 2, 569-579.

Brosius, J., Dull, T. J., Sleeter, D. D. \& Noller, H. F. (1981). Gene 
organization and primary structure of a ribosomal RNA operon from Escherichia coli. J Mol Biol 148, 107-127.

Collins, M. D. \& Stackebrandt, E. (1989). Molecular taxonomic studies on some LL-diaminopimelic acid-containing coryneforms from herbage: description of Nocardioides fastidiosa sp. nov. FEMS Microbiol Lett 57, 289-294.

Collins, M. D., Dorsch, M. \& Stackebrandt, E. (1989). Transfer of Pimelobacter tumescens to Terrabacter gen. nov. as Terrabacter tumescens comb. nov. and of Pimelobacter jensenii to Nocardioides as Nocardioides jensenii comb. nov. Int J Syst Bacteriol 39, 1-6.

Collins, M. D., Cockcroft, S. \& Wallbanks, S. (1994). Phylogenetic analysis of a new LL-diaminopimelic acid-containing coryneform bacterium from herbage, Nocardioides plantarum sp. nov. Int J Syst Bacteriol 44, 523-526.

Dolzani, L., Tonin, E., Lagatolla, C. \& Monti-Bragadin, C. (1994), Typing of Staphylococcus aureus by amplification of the 16S-23S rRNA intergenic spacer sequences. FEMS Microbiol Lett 119, 167-174.

French, J. C., Howells, J. D. \& Anderson, L. E. (1970). US patent 3 551294.

Goodfellow, M. (1989). Genus Rhodococcus Zopf 1891, 28 ${ }^{\mathrm{AL}}$. In Bergey's Manual of Systematic Bacteriology, vol. 4, pp. 23622371. Edited by S. T. Williams, M. E. Sharpe \& J. G. Holt. Baltimore: Williams \& Wilkins.

Gray, P. H. H. \& Thornton, H. G. (1928). Soil bacteria that decompose certain aromatic compounds. Zentbl Bakteriol Parasitenkd Infektkrankh Hyg Abt II 73, 74-96.

Green, C. J. \& Vold, B. S. (1983). Sequence analysis of a cluster of twenty-one tRNA genes in Bacillus subtilis. Nucleic Acids Res $11,5763-5774$.

Green, C. J., Stewart, G. C., Hollis, M. A., Vold, B. S. \& Bott, K. F. (1985). Nucleotide sequence of the Bacillus subtilis ribosomal RNA operon, $r r n B$. Gene 37, 261-266.

Gurtler, V. \& Stanisich, V. A. (1996). New approaches to typing and identification of bacteria using the $16 \mathrm{~S}-23 \mathrm{~S}$ rDNA spacer region. Microbiology 142, 3-16.

Hain, T., Ward-Rainey, N., Kroppenstedt, R. M., Stackebrandt, E. \& Rainey, F. A. (1997). Discrimination of Streptomyces albidoflavus strains based on the size and number of $16 \mathrm{~S}-23 \mathrm{~S}$ ribosomal DNA intergenic spacers. Int $J$ Syst Bacteriol 47, 202-206.

Kim, E., Kim, H., Hong, S.P., Kang, K. H., Kho, Y. H. \& Park, Y.-H. (1993). Gene organization and primary structure of a ribosomal RNA gene cluster from Streptomyces griseus subsp. griseus. Gene 132, 21-31.

Kim, S.-B., Yoon, J.-H., Kim, H., Lee, S.T., Park, Y.-H. \& Goodfellow, M. (1995). A phylogenetic analysis of the genus Saccharomonospora conducted with 16S rRNA gene sequences. Int J Syst Bacteriol 45, 351-356.

Lane, D. J., Pace, B., Olsen, G. J., Stahl, D. A. \& Sogin, M. L. (1985). Rapid determination of $16 \mathrm{~S}$ ribosomal RNA sequences for phylogenetic analyses. Proc Natl Acad Sci USA 82, 6955-6959.

Leblond-Bourget, N., Philippe, H., Mangin, I. \& Decaris, B. (1996). $16 \mathrm{~S}$ rRNA and $16 \mathrm{~S}$ to $23 \mathrm{~S}$ internal transcribed spacer sequence analyses reveal inter- and intraspecific Bifidobacterium phylogeny. Int J Syst Bacteriol 46, 102-111.

Lechevalier, M. P. \& Lechevalier, H. A. (1970). A critical evaluation of the genera of aerobic actinomycetes. In The Actinomycetales, pp. 393-405. Edited by H. Prauser. Jena: Gustav Fischer Verlag.

Lee, S.-T., Rhee, S.-K. \& Lee, G. M. (1994). Biodegradation of pyridine by freely suspended and immobilized Pimelobacter sp. Appl Microbiol Biotechnol 41, 652-657.

Lu, Y. \& Yan, X. (1983). Studies of the classification of thermophilic actinomycetes: determination of thermophilic members of Nocardiaceae. Acta Microbiol Sinica 23, 220-228.

Miller, E. S., Woese, C. R. \& Brenner, S. (1991). Description of the erythromycin-producing bacterium Arthrobacter sp. strain NRRL B-3381 as Aeromicrobium erythreum gen. nov., sp. nov. Int J Syst Bacteriol 41, 363-368.

Navarro, E., Simonet, P., Normand, P. \& Bardin, R. (1992). Characterization of natural populations of Nitrobacter spp. using PCR/RFLP analysis of the ribosomal intergenic spacer. Arch Microbiol 157, 107-115.

Normand, P., Cournoyer, B., Simonet, P. \& Nazaret, S. (1992). Analysis of a ribosomal RNA operon in the actinomycete Frankia. Gene 111, 119-124.

O'Donnell, A. G., Goodfellow, M. \& Minnikin, D. E. (1982). Lipids in the classification of Nocardioides: reclassification of Arthrobacter simplex (Jensen) Lochhead in the genus Nocardioides (Prauser) emend. O'Donnell et al. as Nocardioides simplex comb. nov. Arch Microbiol 133, 323-329.

Pernodet, J.-L., Boccard, F., Alegre, M.-T., Gagnat, J. \& Guérineau, M. (1989). Organization and nucleotide sequence analysis of a ribosomal RNA gene cluster from Streptomyces ambofaciens. Gene 79, 33-46.

Prauser, H. (1976). Nocardioides, a new genus of the order Actinomycetales. Int $J$ Syst Bacteriol 26, 58-65.

Prauser, H. (1984). Nocardioides luteus spec. nov. $Z$ Allg Microbiol 24, 647-648.

Prauser, H. (1989). Genus Nocardioides Prauser 1976, 61 ${ }^{\mathrm{AL}}$. In Bergey's Manual of Systematic Bacteriology, vol. 4, pp. 2371-2375. Edited by S. T. Williams, M. E. Sharpe \& J. G. Holt. Baltimore: Williams \& Wilkins.

Ruan, J.S. \& Zhang, Y. M. (1979). Two new species of Nocardioides. Acta Microbiol Sinica 19, 347-352.

Saitou, N. \& Nei, M. (1987). The neighbor-joining method: a new method for reconstructing phylogenetic trees. Mol Biol Evol 4, $406-425$

Sambrook, J., Fritsch, E. F. \& Maniatis, T. (1989). Molecular Cloning: a Laboratory Manual, 2nd edn, pp. 6.28-6.29. Cold Spring Harbor, NY: Cold Spring Harbor Laboratory.

Stackebrandt, E. \& Goebel, B. M. (1994). Taxonomic note: a place for DNA-DNA reassociation and 16S rRNA sequence analysis in the present species definition in bacteriology. Int $J$ Syst Bacteriol 44, 846-849.

Stackebrandt, E. \& Liesack, W. (1993). Nucleic acids and classification. In Handbook of New Bacterial Systematics, pp. 152-189. Edited by M. Goodfellow \& A. G. O'Donnell. London: Academic Press.

Stackebrandt, E., Rainey, F. A. \& Ward-Rainey, N. L. (1997). Proposal for a new hierarchic classification system, Actinobacteria classis nov. Int J Syst Bacteriol 47, 479-491.

Suzuki, K. \& Komagata, K. (1983). Pimelobacter gen. nov., a new genus of coryneform bacteria with LL-diaminopimelic acid in the cell wall. J Gen Appl Microbiol 29, 59-71.

Suzuki, Y. \& Yamada, T. (1988). The nucleotide sequence of $16 \mathrm{~S}$ rRNA gene from Streptomyces lividans TK21. Nucleic Acids Res 16, 370 .

Suzuki, Y., Ono, Y., Nagata, A. \& Yamada, T. (1988). Molecular cloning and characterization of an rRNA operon in Streptomyces lividans TK21. J Bacteriol 170, 1631-1636. 
Tamura, T. \& Yokota, A. (1994). Transfer of Nocardioides fastidiosa Collins and Stackebrandt 1989 to the genus Aeromicrobium as Aeromicrobium fastidiosum comb. nov. Int J Syst Bacteriol 44, 608-611.

Thompson, J. D., Higgins, D. G. \& Gibson, T. J. (1994). CLUSTAL $\mathrm{W}$ : improving the sensitivity of progressive multiple sequence alignment through sequence weighting, position specific gap penalties and weight matrix choice. Nucleic Acids Res 22, 4673-4680.

Van Wezel, G. P., Vijgenboom, E. \& Bosch, L. (1991). A comparative study of the ribosomal RNA operons of Streptomyces coelicolor A3(2) and sequence analysis of rrnA. Nucleic Acids Res 19, 4399-4403.

Vandamme, P., Pot, B., Gillis, M., De Vos, P., Kersters, K. \& Swings, J. (1996). Polyphasic taxonomy, a consensus approach to bacterial systematics. Microbiol Rev 60, 407-438.

Woese, C. R. (1987). Bacterial evolution. Microbiol Rev 51, 221-271.

Yoon, J.-H., Kim, H., Kim, S.-B., Kim, H.-J., Kim, W. Y., Lee, S. T., Goodfellow, M. \& Park, Y.-H. (1996). Identification of Saccharomonospora strains by the use of genomic DNA fragments and rRNA gene probes. Int J Syst Bacteriol 46, 502-505.

Yoon, J.-H., Rhee, S.-K., Lee, J.-S., Park, Y.-H. \& Lee, S. T. (1997a). Nocardioides pyridinolyticus $\mathrm{sp}$. nov., a pyridine-degrading bacterium isolated from the oxic zone of an oil shale column. Int J Syst Bacteriol 47, 933-938.

Yoon, J.-H., Lee, S. T., Kim, S.-B., Goodfellow, M. \& Park, Y.-H. (1997b). Inter- and intraspecific genetic analysis of the genus Saccharomonospora with $16 \mathrm{~S}$ to $23 \mathrm{~S}$ ribosomal DNA (rDNA) and $23 \mathrm{~S}$ to $5 \mathrm{~S}$ rDNA internally transcribed spacer sequences. Int J Syst Bacteriol 47, 661-669.

Yoon, J.-H., Lee, S. T., Kim, S.-B., Kim, W. Y., Goodfellow, M. \& Park, Y.-H. (1997c). Restriction fragment length polymorphism analysis of PCR-amplified 16S ribosomal DNA for rapid identification of Saccharomonospora strains. Int J Syst Bacteriol 47, 111-114.

Yoon, J.-H., Lee, J.-S., Shin, Y. K., Park, Y.-H. \& Lee, S. T. (1997d). Reclassification of Nocardioides simplex ATCC 13260, ATCC 19565, and ATCC 19566 as Rhodococcus erythropolis. Int J Syst Bacteriol 47, 904-907.

Yoon, J.-H., Lee, S. T. \& Park, Y.-H. (1998). Inter- and intraspecific phylogenetic analysis of the genus Nocardioides and related taxa based on 16S rDNA sequences. Int $J$ Syst Bacteriol 48, 187-194.

Zavaleta, A. I., Martínez-Murcia, A. J. \& Rodriguez-Valera, F. (1996). 16S-23S rDNA intergenic sequences indicate that Leuconostoc oenos is phylogenetically homogeneous. Microbiology 142, 2105-2114. 